\title{
A sufficient condition for the boundedness of operator-weighted martingale transforms and Hilbert transform
}

\author{
by \\ SANDRA PotT (Glasgow)
}

\begin{abstract}
Let $W$ be an operator weight taking values almost everywhere in the bounded positive invertible linear operators on a separable Hilbert space $\mathcal{H}$. We show that if $W$ and its inverse $W^{-1}$ both satisfy a matrix reverse Hölder property introduced by Christ and Goldberg, then the weighted Hilbert transform $H: L_{W}^{2}(\mathbb{R}, \mathcal{H}) \rightarrow L_{W}^{2}(\mathbb{R}, \mathcal{H})$ and also all weighted dyadic martingale transforms $T_{\sigma}: L_{W}^{2}(\mathbb{R}, \mathcal{H}) \rightarrow L_{W}^{2}(\mathbb{R}, \mathcal{H})$ are bounded.

We also show that this condition is not necessary for the boundedness of the weighted Hilbert transform.
\end{abstract}

1. Introduction. The question of finding vector analogues to the celebrated Hunt-Muckenhoupt-Wheeden theorem [9] has been studied intensively in recent years. S. Treil and A. Volberg showed in [13] that a weight function $W$ taking values almost everywhere in the positive invertible $d \times d$ matrices satisfies the vector $A_{2}$ condition

$$
\sup _{I \subset \mathbb{R} \text { interval }}\left\|\langle W\rangle_{I}^{1 / 2}\left\langle W^{-1}\right\rangle_{I}^{1 / 2}\right\|<C
$$

if and only if the Hilbert transform $H$ defines a bounded linear operator on the operator-weighted $L^{2}$-space

$$
L_{W}^{2}\left(\mathbb{R}, \mathbb{C}^{d}\right)=\left\{f: \mathbb{R} \rightarrow \mathbb{C}^{d} \text { measurable }: \int_{\mathbb{R}}\langle W(t) f(t), f(t)\rangle d t<\infty\right\}
$$

or equivalently, if and only if the weighted Hilbert transform

$$
M_{W}^{-1 / 2} H M_{W}^{1 / 2}: L^{2}\left(\mathbb{R}, \mathbb{C}^{d}\right) \rightarrow L^{2}\left(\mathbb{R}, \mathbb{C}^{d}\right)
$$

2000 Mathematics Subject Classification: Primary 42A50, 47B37; Secondary 42A61.

Key words and phrases: operator weight, dyadic martingale transform, weighted Hilbert transform, reverse Hölder property, weighted square function.

The author gratefully acknowledges support by the Nuffield Foundation and the European Network on Analysis and Operators. The author also thanks the Department of Pure Mathematics at the University Bordeaux I, where part of this work was carried out, for their hospitality. 
defines a bounded linear operator. Here, $M_{W}^{1 / 2}$ denotes the densely defined multiplication operator with the matrix function $W^{1 / 2}$, and $\langle\cdot\rangle_{I}$ denotes the average over the interval $I$.

There exists also a dyadic version of this theorem [13]: Let $\mathcal{D}$ denote the collection of all dyadic intervals in $\mathbb{R}$. For each $\sigma \in\{-1,1\}^{\mathcal{D}}$, let $T_{\sigma}$ denote the dyadic martingale transform on $L^{2}(\mathbb{R}, \mathcal{H})$ given by

$$
f \mapsto \sum_{I \in \mathcal{D}} \sigma(I) h_{I} f_{I}
$$

Then a matrix weight function $W$ taking values almost everywhere in the positive invertible $d \times d$ matrices satisfies the vector $A_{2}^{d}$ condition

$$
\sup _{I \in \mathcal{D}}\left\|\langle W\rangle_{I}^{1 / 2}\left\langle W^{-1}\right\rangle_{I}^{1 / 2}\right\|<C
$$

if and only if all weighted dyadic martingale transforms

$$
M_{W}^{-1 / 2} T_{\sigma} M_{W}^{1 / 2}: L^{2}\left(\mathbb{R}, \mathbb{C}^{d}\right) \rightarrow L^{2}\left(\mathbb{R}, \mathbb{C}^{d}\right)
$$

are uniformly bounded.

Characterisations for the boundedness of matrix-weighted Hilbert transforms on $L^{p}\left(\mathbb{R}, \mathbb{C}^{d}\right)$ for $1<p<\infty$ were found in [10], [14].

A different approach via a matrix-weighted Hardy-Littlewood maximal function was suggested more recently by M. Christ and M. Goldberg in [3]. They introduce the matrix reverse Hölder property

$$
\int_{I}\left\|W^{1 / 2}(x)\left\langle W^{-1}\right\rangle_{I}^{1 / 2}\right\|^{r} d x \leq C|I| \quad(I \subset \mathbb{R} \text { interval })
$$

and show that this implies the boundedness of a matrix-weighted maximal function on $L^{p}(\mathbb{R}, \mathcal{H})$ for $p<r$ even in the infinite-dimensional situation, namely when replacing $\mathbb{C}^{d}$ by a separable Hilbert space $\mathcal{H}$, and considering a weight $W: \mathbb{R} \rightarrow \mathcal{L}(\mathcal{H})$ taking values almost everywhere in the bounded positive invertible linear operators on $\mathcal{H}$. It was shown in [8] that in the finitedimensional situation, the boundedness of the weighted maximal function in turn implies the boundedness of the weighted Hilbert transform.

In the finite-dimensional situation, the vector $A_{2}$ condition implies the matrix reverse Hölder property for some $r>2$ [3], so that this approach provides a new proof of the above mentioned result in [13].

The characterisation of the boundedness of the weighted Hilbert transform and martingale transforms in the infinite-dimensional setting has proved very difficult, even in the case $p=2$. It was shown in [4]-[6] that the operator versions of (1) and (2) are not sufficient for the boundedness of the weighted Hilbert transform and the weighted dyadic martingale transforms, respectively ((1) and (2) were shown to be necessary in [13]). 
In this paper, we show that even in the infinite-dimensional situation, the matrix reverse Hölder condition (3) for an operator weight $W$ and its inverse $W^{-1}$ for some $r>2$ implies the uniform boundedness of all weighted dyadic martingale transforms and the boundedness of the weighted Hilbert transform on $L^{2}(\mathbb{R}, \mathcal{H})$. We use a slightly different route from [3], [8]. Instead of using the weighted maximal function, we show first that the weighted square function operator is bounded and bounded below by means of a stopping time argument from [11]. This gives us the uniform boundedness of the weighted dyadic martingale transforms. The case of the Hilbert transform then follows from a result in [12].

Using the theory of vector BMO functions, we also show that the reverse Hölder property is not necessary in the infinite-dimensional case, even with $r=2$.

\section{Weights with reverse Hölder property and decaying stopping time}

Definition 2.1 ([3]). We say that an operator weight $W: \mathbb{R} \rightarrow \mathcal{L}(\mathcal{H})$ has the matrix reverse Hölder property if there exist constants $C>0$ and $r>2$ such that

$$
\int_{I}\left\|W^{1 / 2}(x)\left\langle W^{-1}\right\rangle_{I}^{1 / 2}\right\|^{r} d x \leq C|I|
$$

for all intervals $I \subset \mathbb{R}$.

Definition 2.2. We say that an operator weight $W: \mathbb{R} \rightarrow \mathcal{L}(\mathcal{H})$ has the dyadic matrix reverse Hölder property if there exist constants $C>0$ and $r>2$ such that

$$
\int_{I}\left\|W^{1 / 2}(x)\left\langle W^{-1}\right\rangle_{I}^{1 / 2}\right\|^{r} d x \leq C|I|
$$

for all dyadic intervals $I \in \mathcal{D}$.

Such weights satisfy in particular the (dyadic) vector $A_{2}$ condition (1), (2). Furthermore, for each operator-valued weight and each interval $I \subseteq \mathbb{R}$, one has the elementary inequality

$$
\left\|\langle W\rangle_{I}^{-1 / 2}\left\langle W^{-1}\right\rangle_{I}^{-1 / 2}\right\| \leq 1
$$

(see [13], [3]).

For each $I \in \mathcal{D}$, let $\mathcal{D}(I)$ denote the collection of all $J \in \mathcal{D}$ with $J \subseteq I$.

Given $I \in \mathcal{D}$ and $\lambda>1$, let $\mathcal{J}_{\lambda, 1}(I)$ denote the collection of all maximal dyadic subintervals $I_{\lambda}$ of $I$ such that

$$
\left\|\frac{1}{\left|I_{\lambda}\right|} \int_{I_{\lambda}}\left\langle W^{-1}\right\rangle_{I}^{1 / 2} W(x)\left\langle W^{-1}\right\rangle_{I}^{1 / 2} d x\right\|>\lambda
$$


or

$$
\left\|\frac{1}{\left|I_{\lambda}\right|} \int_{I_{\lambda}}\langle W\rangle_{I}^{1 / 2} W^{-1}(x)\langle W\rangle_{I}^{1 / 2} d x\right\|>\lambda
$$

We write $\mathcal{J}_{\lambda, 0}(I)=\{I\}, \mathcal{J}_{\lambda, k}(I)=\bigcup_{J \in \mathcal{J}_{\lambda, k-1}(I)} \mathcal{J}(J)$ for $k \geq 1, \mathcal{F}(I)=$ $\mathcal{F}_{\lambda, 1}(I)=\mathcal{D}(I) \backslash \bigcup_{J \in \mathcal{J}(I)} \mathcal{D}(J)$, and $\mathcal{F}_{\lambda, k}(I)=\bigcup_{J \in \mathcal{J}_{\lambda, k-1}(I)} \mathcal{F}(J)$ for $k \geq 1$.

Note that with this notation, for each $I \in \mathcal{D}, \mathcal{D}(I)$ is the disjoint union of the $\mathcal{F}_{\lambda, k}(I), k \in \mathbb{N}$. Somewhat loosely, we will write $\bigcup \mathcal{J}(I)$ for the set $\bigcup_{J \in \mathcal{J}(I)} J \subseteq I$ and $|\mathcal{J}(I)|$ for $\left|\bigcup_{J \in \mathcal{J}(I)} J\right|$.

LEMMA 2.3. If $W$ and $W^{-1}$ both have the dyadic matrix reverse Hölder property, then for sufficiently large $\lambda$, there exists a constant $c, 0<c<1$, such that

$$
\left|\mathcal{J}_{\lambda, k}(I)\right| \leq c^{k}|I| \quad \text { for all } I \in \mathcal{D}, k \in \mathbb{N}
$$

(i.e. $\mathcal{J}_{\lambda}(I)$ is a decaying stopping time in the sense of $[11$, Section 3.3$\left.]\right)$.

Proof. The proof is an adaptation of the proof of the Weight Lemma 3.17 for scalar weights in [11].

We first introduce an auxiliary stopping time $\mathcal{G}$. For $I \in \mathcal{D}$, let $\mathcal{G}(I)$ denote the collection of all maximal dyadic subintervals $J$ of $I$ such that (5) holds. We show that $\mathcal{G}$ is a decaying stopping time for sufficiently large $\lambda$.

Note that

$$
\left\langle W^{-1}\right\rangle_{I}^{1 / 2} W(x)\left\langle W^{-1}\right\rangle_{I}^{1 / 2} \leq \lambda \mathbf{1}_{\mathcal{H}}
$$

in the sense of an operator inequality almost everywhere on $G^{I}=I \backslash \bigcup \mathcal{G}(I)$. Therefore,

$$
\begin{aligned}
\int_{G^{I}} W(x) d x & =\left\langle W^{-1}\right\rangle_{I}^{-1 / 2}\left(\int_{G^{I}}\left\langle W^{-1}\right\rangle_{I}^{1 / 2} W(x)\left\langle W^{-1}\right\rangle_{I}^{1 / 2} d x\right)\left\langle W^{-1}\right\rangle_{I}^{-1 / 2} \\
& \leq \lambda\left|G^{I}\right|\left\langle W^{-1}\right\rangle_{I}^{-1} \leq \lambda\left|G^{I}\right|\langle W\rangle_{I}
\end{aligned}
$$

by (4).

It is enough to show that there exists a constant $\alpha>0$ (independent of $I$ ) such that $\left|G^{I}\right| \geq \alpha|I|$. Assume towards a contradiction that this is false. Then there exists an interval $I \in \mathcal{D}$ such that $\left|G^{I}\right| \leq|I| / 2 \lambda$ and

$$
\int_{G^{I}} W(x) d x \leq \frac{1}{2}\langle W\rangle_{I}|I| .
$$

Let $\mathcal{G}(I)=\left\{I_{\lambda, j}\right\}_{j}$. Then 


$$
\sum_{j} \int_{I_{\lambda, j}} W(x) d x=|I|\langle W\rangle_{I}-\int_{G^{I}} W(x) d x \geq \frac{1}{2}|I|\langle W\rangle_{I} .
$$

However, one also has

$$
\begin{array}{rl}
\sum_{j} \int_{I_{\lambda, j}} & W(x) d x \\
& =\left\langle W^{-1}\right\rangle_{I}^{-1 / 2}\left(\sum_{j} \int_{I_{\lambda, j}}\left\langle W^{-1}\right\rangle_{I}^{1 / 2} W(x)\left\langle W^{-1}\right\rangle_{I}^{1 / 2} d x\right)\left\langle W^{-1}\right\rangle_{I}^{-1 / 2} \\
& \leq 2 \lambda\left\langle W^{-1}\right\rangle_{I}^{-1} \sum_{j}\left|I_{\lambda, j}\right| \leq 2 \lambda\langle W\rangle_{I} \sum_{j}\left|I_{\lambda, j}\right|
\end{array}
$$

and therefore

$$
\sum_{j}\left|I_{\lambda, j}\right| \geq \frac{1}{4 \lambda}|I|
$$

By the dyadic matrix reverse Hölder property, for some $p>1$ we have

$$
\begin{aligned}
C|I| & \geq \int_{I}\left\|\left\langle W^{-1}\right\rangle_{I}^{1 / 2} W(x)\left\langle W^{-1}\right\rangle_{I}^{1 / 2}\right\|^{p} d x \\
& \geq \sum_{j} \int_{I_{\lambda, j}}\left\|\left\langle W^{-1}\right\rangle_{I}^{1 / 2} W(x)\left\langle W^{-1}\right\rangle_{I}^{1 / 2}\right\|^{p} d x \\
& \geq \sum_{j} \frac{1}{\left|I_{\lambda, j}\right|^{p-1}}\left(\int_{I_{\lambda, j}}\left\|\left\langle W^{-1}\right\rangle_{I}^{1 / 2} W(x)\left\langle W^{-1}\right\rangle_{I}^{1 / 2}\right\| d x\right)^{p} \geq \sum_{j}\left|I_{\lambda, j}\right| \lambda^{p} .
\end{aligned}
$$

Thus

$$
\sum_{j}\left|I_{\lambda, j}\right| \leq \frac{C}{\lambda^{p}}|I| .
$$

Choosing $\lambda \geq(4 C)^{1 / p-1}$, we obtain a contradiction to (10). This proves that for $\lambda \geq(4 C)^{1 / p-1}$, there exists a constant $0<c^{\prime}<1$ such that $|\mathcal{G}(J)| \leq c^{\prime}|J|$ for all $J \in \mathcal{D}$. It then follows by induction that $\left|\mathcal{G}^{k}(J)\right| \leq c^{\prime k}|J|$ for $k \in \mathbb{N}$ and $J \in \mathcal{D}$.

Let us now write $\mathcal{G}_{\lambda}(J)$ to indicate which $\lambda$ we are using in the definition of our stopping time. Notice that for each interval $K \in \mathcal{G}_{2 \lambda^{2}}(J)$, there exists $\widetilde{K} \in \mathcal{G}_{\lambda}^{2}(J)$ with $K \subseteq \widetilde{K}$. To see this, let $L \subset J$ be the unique dyadic interval in $\mathcal{G}_{\lambda}(J)$ such that $K \subseteq L$. Writing

$$
\begin{aligned}
& \int_{K}\left\langle W^{-1}\right\rangle_{J}^{1 / 2} W(x)\left\langle W^{-1}\right\rangle_{J}^{1 / 2} d x \\
& \quad=\left\langle W^{-1}\right\rangle_{J}^{1 / 2}\left\langle W^{-1}\right\rangle_{L}^{-1 / 2} \int_{K}\left\langle W^{-1}\right\rangle_{L}^{1 / 2} W(x)\left\langle W^{-1}\right\rangle_{L}^{1 / 2} d x\left\langle W^{-1}\right\rangle_{L}^{-1 / 2}\left\langle W^{-1}\right\rangle_{J}^{1 / 2},
\end{aligned}
$$


we obtain

$$
\begin{aligned}
2 \lambda^{2} & <\left\|\left\langle W^{-1}\right\rangle_{J}^{1 / 2}\langle W\rangle_{K}\left\langle W^{-1}\right\rangle_{J}^{1 / 2}\right\| \\
& \leq\left\|\left\langle W^{-1}\right\rangle_{J}^{1 / 2}\left\langle W^{-1}\right\rangle_{L}^{-1}\left\langle W^{-1}\right\rangle_{J}^{1 / 2}\right\|\left\|\frac{1}{|K|} \int_{K}\left\langle W^{-1}\right\rangle_{L}^{1 / 2} W(x)\left\langle W^{-1}\right\rangle_{L}^{1 / 2} d x\right\| \\
& \leq\left\|\left\langle W^{-1}\right\rangle_{J}^{1 / 2}\langle W\rangle_{L}\left\langle W^{-1}\right\rangle_{J}^{1 / 2}\right\|\left\|\frac{1}{|K|} \int_{K}\left\langle W^{-1}\right\rangle_{L}^{1 / 2} W(x)\left\langle W^{-1}\right\rangle_{L}^{1 / 2} d x\right\| \\
& \leq 2 \lambda\left\|\frac{1}{|K|} \int_{K}\left\langle W^{-1}\right\rangle_{L}^{1 / 2} W(x)\left\langle W^{-1}\right\rangle_{L}^{1 / 2} d x\right\|
\end{aligned}
$$

by (4), which yields

$$
\left\|\frac{1}{|K|} \int_{K}\left\langle W^{-1}\right\rangle_{L}^{1 / 2} W(x)\left\langle W^{-1}\right\rangle_{L}^{1 / 2} d x\right\|>\lambda
$$

Consequently, there exists $\widetilde{K} \supseteq K$ such that $\widetilde{K} \in \mathcal{J}_{\lambda}(L) \subset \mathcal{J}_{\lambda, 2}(J)$. Therefore $\left|\mathcal{G}_{2 \lambda^{2}}(J)\right| \leq\left|\mathcal{G}_{\lambda}^{2}(J)\right| \leq c^{\prime 2}|J|$. By iteration, it follows that by choosing $\lambda$ sufficiently large, we can assume that $\left|\mathcal{G}_{\lambda}(J)\right| \leq \frac{1}{4}|J|$ for $J \in \mathcal{D}$.

We now define a second auxiliary stopping time $\widetilde{\mathcal{G}}$. For $J \in \mathcal{D}$, let $\widetilde{\mathcal{G}}(J)$ be the collection of all maximal dyadic subintervals of $J$ such that (6) holds.

Using now the reverse Hölder property of $W^{-1}$, we find that also $\widetilde{\mathcal{G}}$ is a decaying stopping time for sufficiently large $\lambda$. Again, by choosing $\lambda$ large enough, we can assume that $\left|\widetilde{\mathcal{G}}_{\lambda}(J)\right| \leq \frac{1}{4}|J|$ for $J \in \mathcal{D}$.

This means that for $\lambda$ large enough,

$$
\left|\mathcal{J}_{\lambda}(J)\right| \leq\left|\mathcal{G}_{\lambda}(J)\right|+\left|\widetilde{\mathcal{G}}_{\lambda}(J)\right| \leq \frac{1}{2}|J| \quad(J \in \mathcal{D}) .
$$

Thus $\mathcal{J}_{\lambda}$ is a decaying stopping time.

\section{The weighted square function}

THEOREM 3.1. Let $W: \mathbb{R} \rightarrow \mathcal{L}(\mathcal{H})$ be an operator weight such that $W$ and $W^{-1}$ both have the dyadic matrix reverse Hölder property. Then the weighted square function operator

$$
S_{W}: L^{2}(\mathbb{R}, \mathcal{H}) \rightarrow L^{2}(\mathbb{R}, \mathcal{H}), \quad f \mapsto \sum_{I \in \mathcal{D}}\left\langle W^{-1}\right\rangle_{I}^{1 / 2}\left(W^{1 / 2} f\right)_{I} h_{I}(x)
$$

is bounded and invertible. 
Proof. We will first show that the operator

$$
T: L^{2}(\mathbb{R}, \mathcal{H}) \rightarrow L^{2}(\mathbb{R}, \mathcal{H}), \quad f \mapsto \sum_{I \in \mathcal{D}} W^{1 / 2}(x)\langle W\rangle_{I}^{-1 / 2} f_{I} h_{I}(x),
$$

is bounded, following the steps of the proof of Theorem 6.1 in [11].

We choose $\lambda>1$ such that $\mathcal{J}_{\lambda}(I)$ is a decaying stopping time, and write just $\mathcal{J}(I)$. First note that for almost every $x \in J \backslash \bigcup \mathcal{J}(J)$,

$$
\left\langle W^{-1}\right\rangle_{J}^{1 / 2} W(x)\left\langle W^{-1}\right\rangle_{J}^{1 / 2} \leq \lambda \mathbf{1}_{\mathcal{H}}
$$

and

$$
\langle W\rangle_{J}^{1 / 2} W^{-1}(x)\langle W\rangle_{J}^{1 / 2} \leq \lambda \mathbf{1}_{\mathcal{H}},
$$

therefore

$$
\frac{1}{\lambda} \mathbf{1}_{\mathcal{H}} \leq\left\langle W^{-1}\right\rangle_{J}^{1 / 2} W(x)\left\langle W^{-1}\right\rangle_{J}^{1 / 2} \leq \lambda \mathbf{1}_{\mathcal{H}}
$$

by (4). Let $f \in L^{2}(\mathbb{R}, \mathcal{H})$ have finite Haar expansion. We can assume without loss of generality that $f$ is supported in $[0,1]$ and has mean 0 . We write $\mathcal{J}_{k}$ for $\mathcal{J}_{k}([0,1])$, and $\mathcal{F}_{k}$ for $\mathcal{F}_{k}([0,1])$. For $j \in \mathbb{N}$, write

$$
\begin{gathered}
\Delta_{j} f=\sum_{K \in \mathcal{F}_{j}} h_{K} f_{K}=\sum_{I \in \mathcal{J}_{j-1}} \sum_{J \in \mathcal{F}(I)} f_{J} h_{J}=\sum_{I \in \mathcal{J}_{j-1}} \Delta_{I} f \\
M_{j} f=\sum_{J \in \mathcal{F}_{j}}\langle W\rangle_{J}^{-1 / 2} f_{J} h_{J}=\sum_{I \in \mathcal{J}_{j-1}} \sum_{J \in \mathcal{F}(I)}\langle W\rangle_{J}^{-1 / 2} f_{J} h_{J}=\sum_{I \in \mathcal{J}_{j-1}} M_{I} f
\end{gathered}
$$

and

$$
\begin{aligned}
T_{j} f=T \Delta_{j} f=W^{1 / 2}(x) M_{j} f & =\sum_{I \in \mathcal{J}_{j-1}} W^{1 / 2}(x) \sum_{J \in \mathcal{F}(I)}\langle W\rangle_{J}^{-1 / 2} f_{J} h_{J} \\
& =\sum_{I \in \mathcal{J}_{j-1}} W^{1 / 2}(x) M_{I} f .
\end{aligned}
$$

Then $\sum_{j=1}^{\infty} \Delta_{j} f=f$ and $\sum_{j=1}^{\infty} T_{j} f=T f$. We will show that the $T_{j}$ satisfy the conditions of Cotlar's lemma.

Each $M_{I} f$ has support in $I$, so $T_{j} f$ has support on the disjoint intervals in $\mathcal{J}_{j-1}$. We write

$$
\left\|T_{j} f\right\|^{2}=\int_{\cup \mathcal{J}_{j-1}}\left\|T_{j} f\right\|^{2} d x=\int_{\cup \mathcal{J}_{j-1} \backslash \cup \mathcal{J}_{j}}\left\|T_{j} f\right\|^{2} d x+\int_{\cup \mathcal{J}_{j}}\left\|T_{j} f\right\|^{2} d x
$$

and estimate the terms separately. 
First note that

$$
\begin{aligned}
& \int_{\cup \mathcal{J}_{j-1} \backslash \cup \mathcal{J}_{j}}\left\|T_{j} f\right\|^{2} d x=\sum_{J \in \mathcal{J}_{j-1}} \int_{J \backslash \cup \mathcal{J}(J)}\left\|T_{j} f\right\|^{2} d x \\
&= \sum_{J \in \mathcal{J}_{j-1}} \int_{J \backslash \cup \mathcal{J}(J)}\left\|W^{1 / 2}(x)\left\langle W^{-1}\right\rangle_{J}^{1 / 2}\left\langle W^{-1}\right\rangle_{J}^{-1 / 2} M_{J} f\right\|^{2} d x \\
& \leq \sum_{J \in \mathcal{J}_{j-1}} \int_{J \backslash \cup \mathcal{J}(J)}\left\|W^{1 / 2}(x)\left\langle W^{-1}\right\rangle_{J}^{1 / 2}\right\|^{2}\left\|\left\langle W^{-1}\right\rangle_{J}^{-1 / 2} M_{J} f\right\|^{2} d x \\
& \leq \lambda \sum_{J \in \mathcal{J}_{j-1}} \int_{J \backslash \cup \mathcal{J}(J)}\left\|\left\langle W^{-1}\right\rangle_{J}^{-1 / 2} M_{J} f\right\|^{2} d x .
\end{aligned}
$$

Note that for each $J \in \mathcal{J}_{j-1}$,

$$
\begin{aligned}
\left\|\left\langle W^{-1}\right\rangle_{J}^{-1 / 2} M_{J} f\right\|^{2} & =\left\|\left\langle W^{-1}\right\rangle_{J}^{-1 / 2} \sum_{K \in \mathcal{F}(J)}\langle W\rangle_{K}^{-1 / 2} f_{K} h_{K}\right\|^{2} \\
& \leq C \sum_{K \in \mathcal{F}(J)}\left\|\langle W\rangle_{J}^{1 / 2}\left\langle W^{-1}\right\rangle_{K}^{1 / 2}\right\|^{2}\left\|f_{K}\right\|^{2} \leq C \lambda\left\|\Delta_{J} f\right\|^{2},
\end{aligned}
$$

since for $K \in \mathcal{F}(J)$, one has

$$
\langle W\rangle_{J}^{1 / 2}\left\langle W^{-1}\right\rangle_{K}\langle W\rangle_{J}^{1 / 2}=\frac{1}{|K|} \int_{K}\langle W\rangle_{J}^{1 / 2} W^{-1}(x)\langle W\rangle_{J}^{1 / 2} d x \leq \lambda .
$$

Using the disjointness of the $J \in \mathcal{J}_{j-1}$, we obtain

$$
\int_{\cup \mathcal{J}_{j-1} \backslash \cup \mathcal{J}_{j}}\left\|T_{j} f\right\|^{2} d x \leq C \lambda^{2}\left\|\Delta_{j} f\right\|^{2} .
$$

Now we consider the other part:

$$
\begin{aligned}
& \int_{\cup \mathcal{J}_{j}}\left\|T_{j} f\right\|^{2} d x=\sum_{J \in \mathcal{J}_{j-1}} \sum_{I \in \mathcal{J}(J) I} \int_{I}\left\|W^{1 / 2}(x) \sum_{K \in \mathcal{F}(J)}\langle W\rangle_{K}^{-1 / 2} f_{K} h_{K}\right\|^{2} d x \\
&=\sum_{J \in \mathcal{J}_{j-1}} \sum_{I \in \mathcal{J}(J)} \int_{I}\left\|\langle W\rangle_{I}^{1 / 2} \sum_{K \in \mathcal{F}(J)}\langle W\rangle_{K}^{-1 / 2} f_{K} h_{K}\right\|^{2} d x \\
& \leq \sum_{J \in \mathcal{J}_{j-1}} \sum_{I \in \mathcal{J}(J)}\left\|\langle W\rangle_{I}^{1 / 2}\langle W\rangle_{J}^{-1 / 2}\right\|^{2} \int_{I}\left\|\langle W\rangle_{J}^{1 / 2} \sum_{K \in \mathcal{F}(J)}\langle W\rangle_{K}^{-1 / 2} f_{K} h_{K}\right\|^{2} d x \\
& \leq 2 \lambda^{2} \sum_{J \in \mathcal{J}_{j-1}}\left\|\Delta_{J} f\right\|^{2}=2 \lambda^{2}\left\|\Delta_{j} f\right\|^{2} .
\end{aligned}
$$

Here, we use that for $K \in \mathcal{F}(J), h_{K}$ is constant on each $I \in \mathcal{J}(J)$. Altogether, there exists a constant $A>0$ such that $\left\|T_{j} f\right\|^{2} \leq A\left\|\Delta_{j} f\right\|^{2}$. We will 
now show that there exist $0<d<1$ and a constant $\widetilde{A}>0$ such that for any $k>j$,

$$
\int_{\cup \mathcal{J}_{k-1}}\left\|T_{j} f\right\|^{2} d x \leq \widetilde{A} d^{k-j}\left\|\Delta_{j} f\right\|^{2}
$$

Note that $M_{j} f$ is constant on each $J \in \mathcal{J}_{j}$. We write $M_{j} f(J)$ to indicate the value of $M_{j} f$ on such an interval $J$. Thus

$$
\begin{aligned}
& \int_{\cup}\left\|T_{j} f\right\|^{2} d x=\sum_{J \in \mathcal{J}_{j}} \sum_{I \in \mathcal{J}_{k-j-1}(J) I} \int_{I}\left\|W^{1 / 2}(x) M_{j} f(J)\right\|^{2} d x \\
= & \sum_{J \in \mathcal{J}_{j}} \sum_{I \in \mathcal{J}_{k-j-1}(J) I}\left\|\langle W\rangle_{I}^{1 / 2} M_{j} f(J)\right\|^{2} d x \\
= & \sum_{J \in \mathcal{J}_{j}} \sum_{I \in \mathcal{J}_{k-j-1}(J)}\left\|\langle W\rangle_{I}^{1 / 2} M_{j} f(J)\right\|^{2}|I| \\
= & \sum_{J \in \mathcal{J}_{j}}\left\langle\sum_{I \in \mathcal{J}_{k-j-1}(J)}|I|\langle W\rangle_{I} M_{j} f(J), M_{j} f(J)\right\rangle \\
\leq & \sum_{\widetilde{J} \in \mathcal{J}_{j-1}} \sum_{J \in \mathcal{J}(\widetilde{J})}\left\|\left\langle W^{-1}\right\rangle_{J}^{1 / 2}\left(\sum_{I \in \mathcal{J}_{k-j-1}(J)}|I|\langle W\rangle_{I}\right)\left\langle W^{-1}\right\rangle_{J}^{1 / 2}\right\| \\
& \times\left\|\left\langle W^{-1}\right\rangle_{J}^{-1 / 2}\langle W\rangle_{\widetilde{J}}^{-1 / 2}\right\|^{2}\left\|\langle W\rangle_{\widetilde{J}}^{1 / 2} M_{j} f(J)\right\|^{2} \\
\leq & \sum_{\widetilde{J} \in \mathcal{J}_{j-1}} \sum_{J \in \mathcal{J}(\widetilde{J})}\left\|\sum_{I \in \mathcal{J}_{k-j-1}(J)}\left\langle W^{-1}\right\rangle_{J}^{1 / 2}|I|\langle W\rangle_{I}\left\langle W^{-1}\right\rangle_{J}^{1 / 2}\right\| \\
& \times\left\|\langle W\rangle_{J}^{1 / 2}\langle W\rangle_{\widetilde{J}}^{-1 / 2}\right\|^{2}\left\|\langle W\rangle_{\widetilde{J}}^{1 / 2} M_{j} f(J)\right\|^{2} \\
\leq & 2 \lambda \sum_{\widetilde{J} \in \mathcal{J}_{j-1}} \sum_{J \in \mathcal{J}(\widetilde{J})} \int_{U}\left\|\left\langle W^{-1}\right\rangle_{J}^{1 / 2} W(x)\left\langle W^{-1}\right\rangle_{J}^{1 / 2}\right\| d x\left\|\langle W\rangle_{\widetilde{J}}^{1 / 2} M_{j} f(J)\right\|^{2} .
\end{aligned}
$$

Now we employ first Hölder's inequality, the reverse Hölder property (2.1) and Lemma 2.3 to continue with the estimate:

$$
\begin{aligned}
& \int_{\cup \mathcal{J}_{k-1}}\left\|T_{j} f\right\|^{2} d x \\
& \leq 2 \lambda \sum_{\widetilde{J} \in \mathcal{J}_{j-1}} \sum_{J \in \mathcal{J}(\widetilde{J})}\left(\int_{\cup \mathcal{J}_{k-j-1}(J)}\left\|\left\langle W^{-1}\right\rangle_{J}^{1 / 2} W^{1 / 2}(x)\right\|^{2 p} d x\right)^{1 / p} \\
& \times\left|\mathcal{J}_{k-j-1}(J)\right|^{1 / p^{\prime}}\left\|\langle W\rangle_{\widetilde{J}}^{1 / 2} M_{j} f(J)\right\|^{2}
\end{aligned}
$$




$$
\begin{aligned}
& \leq 2 \lambda \sum_{\widetilde{J} \in \mathcal{J}_{j-1}} \sum_{J \in \mathcal{J}(\widetilde{J})}\left(\int_{\cup \mathcal{J}_{k-j-1}(J)}\left\|\left\langle W^{-1}\right\rangle_{J}^{1 / 2} W^{1 / 2}(x)\right\|^{2 p} d x\right)^{1 / p} \\
& \times c^{(k-j-1) / p^{\prime}}|J|^{1-1 / p}\left\|\langle W\rangle_{\widetilde{J}}^{1 / 2} M_{j} f(J)\right\|^{2} \\
& \leq 2 \lambda C^{1 / p}\left(c^{1 / p^{\prime}}\right)^{k-j-1} \sum_{\widetilde{J} \in \mathcal{J}_{j-1}} \sum_{J \in \mathcal{J}(\widetilde{J})}|J|\left\|\langle W\rangle_{\widetilde{J}}^{1 / 2} M_{j} f(J)\right\|^{2} \\
& =2 \lambda C^{1 / p}\left(c^{1 / p^{\prime}}\right)^{k-j-1} \sum_{\widetilde{J} \in \mathcal{J}_{j-1} \cup \mathcal{J}(\widetilde{J})}\left\|T_{j} f\right\|^{2} d x \\
& \leq C^{1 / p} 4 \lambda^{3}\left(c^{1 / p^{\prime}}\right)^{k-j-1}\left\|\Delta_{j} f\right\|^{2}
\end{aligned}
$$

by (16). Here, $p=r / 2>1$, and $1 / p+1 / p^{\prime}=1$. Choosing $d=c^{1 / p^{\prime}}$, we obtain the statement.

Boundedness of $T$ now follows from Cotlar's lemma (see e.g. [11, 2.4]), since $T_{k} f$ is supported on $\bigcup \mathcal{J}_{k-1}$, and the $T_{k}^{*}$ have orthogonal ranges.

It remains to be shown that $S_{W}$ is bounded. Let $M_{W}^{1 / 2}$ be the densely defined multiplication operator with the operator function $W^{1 / 2}$ on $L^{2}(\mathbb{R}, \mathcal{H})$, let $D_{W}$ be the densely defined operator on $L^{2}(\mathbb{R}, \mathcal{H})$ which is given by $D_{W} e h_{I}=\langle W\rangle_{I}^{1 / 2} e h_{I}$ for each $I \in \mathcal{D}$ and $e \in \mathcal{H}$, let $D_{W}^{-1}$ be the densely defined operator on $L^{2}(\mathbb{R}, \mathcal{H})$ given by $D_{W}^{-1} e h_{I}=\langle W\rangle_{I}^{-1 / 2} e h_{I}$ for $I \in \mathcal{D}$, $e \in \mathcal{H}$, and let $D_{W^{-1}}, D_{W^{-1}}^{-1}$ be defined correspondingly.

With this notation, we have shown that $T=M_{W}^{1 / 2} D_{W}^{-1}$ extends to a bounded linear operator on $L^{2}(\mathbb{R}, \mathcal{H})$. Since $W^{-1}$ satisfies the vector $A_{2}$ condition, $D_{W^{-1}} D_{W}$ defines a bounded invertible linear operator on $L^{2}(\mathbb{R}, \mathcal{H})$. It follows that $S_{W}=D_{W^{-1}} M_{W}^{1 / 2}$ is a bounded linear operator on $L^{2}(\mathbb{R}, \mathcal{H})$ and has a bounded inverse $S_{W}^{-1}=M_{W^{-1}}^{1 / 2} D_{W^{-1}}^{-1}=S_{W^{-1}}^{*} D_{W}^{-1} D_{W^{-1}}^{-1}$.

\section{Boundedness of the weighted dyadic martingale transforms} and of the weighted Hilbert transform. We can now prove our main result.

Theorem 4.1. Let $W: \mathbb{R} \rightarrow \mathcal{L}(\mathcal{H})$ be an operator weight.

(1) Suppose that $W$ and $W^{-1}$ both have the dyadic matrix reverse Hölder property. Then for each $\sigma \in\{-1,1\}^{\mathcal{D}}$, the weighted martingale transform $M_{W}^{-1 / 2} T_{\sigma} M_{W}^{1 / 2}$ defines a bounded invertible linear operator on $L^{2}(\mathbb{R}, \mathcal{H})$, and the norms of these operators are uniformly bounded.

(2) Suppose that $W$ and $W^{-1}$ both have the matrix reverse Hölder property. Then the weighted Hilbert transform $M_{W}^{-1 / 2} H M_{W}^{1 / 2}$ defines a bounded invertible linear operator on $L^{2}(\mathbb{R}, \mathcal{H})$. 
Proof. It suffices to show (1), since the case of the Hilbert transform then follows from [12] together with the fact that the matrix reverse Hölder property 2.1 implies the dyadic matrix reverse Hölder property 2.2 for all translations and dilations of $W$ and $W^{-1}$.

By Theorem 3.1,

$$
M_{W}^{-1 / 2} T_{\sigma} M_{W}^{1 / 2}=M_{W}^{-1 / 2} D_{W^{-1}}^{-1} T_{\sigma} D_{W^{-1}} M_{W}^{1 / 2}=S_{W}^{-1} T_{\sigma} S_{W}
$$

extends to a bounded invertible operator on $L^{2}(\mathbb{R}, \mathcal{H})$ for each $\sigma \in\{-1,1\}^{\mathcal{D}}$.

\section{A counterexample to the necessity of the reverse Hölder property}

THEOREM 5.1. There exist an infinite-dimensional Hilbert space $\mathcal{K}$ and an operator weight $W: \mathbb{R} \rightarrow \mathcal{L}(\mathcal{K})$ such that the weighted Hilbert transform $M_{W}^{1 / 2} H M_{W}^{-1 / 2}: L^{2}(\mathbb{R}, \mathcal{K}) \rightarrow L^{2}(\mathbb{R}, \mathcal{K})$ is bounded, but the matrix reverse Hölder condition 2.1 does not hold for $W$.

Proof. Let $\mathcal{H}$ be an infinite-dimensional separable Hilbert space, and consider an operator weight of the form

(18) $\quad W: \mathbb{R} \rightarrow \mathcal{L}(\mathcal{H} \oplus \mathcal{H}), W(t)=V(t)^{*} V(t)$, where $V(t)=\left(\begin{array}{cc}\mathbf{1}_{\mathcal{H}} & B(t) \\ 0 & \mathbf{1}_{\mathcal{H}}\end{array}\right)$, where $B$ is a weakly integrable $\mathcal{L}(\mathcal{H})$-valued function.

An easy calculation (see [7], [4]) shows that then the Hilbert transform defines a bounded linear operator $L_{W}^{2}(\mathbb{R}, \mathcal{H} \oplus \mathcal{H}) \rightarrow L_{W}^{2}(\mathbb{R}, \mathcal{H} \oplus \mathcal{H})$ if and only if the commutator $[H, B]$ defines a bounded linear operator $L^{2}(\mathbb{R}, \mathcal{H}) \rightarrow$ $L^{2}(\mathbb{R}, \mathcal{H})$.

Now notice that for each interval $I \subset \mathbb{R}$ and each $x \in I$,

$$
\begin{aligned}
& \left\|\left\langle W^{-1}\right\rangle_{I}^{1 / 2} W(x)\left\langle W^{-1}\right\rangle_{I}^{1 / 2}\right\| \\
& \quad=\varrho\left(\left\langle W^{-1}\right\rangle_{I} W(x)\right)=\varrho\left(\left\langle V^{-1} V^{*-1}\right\rangle_{I} V^{*}(x) V(x)\right) \\
& \quad=\varrho\left(V(x)\left\langle V^{-1} V^{*-1}\right\rangle_{I} V^{*}(x)\right)=\left\|V(x)\left\langle V^{-1} V^{*-1}\right\rangle_{I} V^{*}(x)\right\|
\end{aligned}
$$

and

$$
\begin{aligned}
& V(x)\left\langle V^{-1} V^{*-1}\right\rangle_{I} V^{*}(x) \\
&=\left(\begin{array}{cc}
\mathbf{1}_{\mathcal{H}} & B(x) \\
0 & \mathbf{1}_{\mathcal{H}}
\end{array}\right)\left(\begin{array}{cc}
\mathbf{1}_{\mathcal{H}}+\left\langle B B^{*}\right\rangle_{I} & -\langle B\rangle_{I} \\
-\left\langle B^{*}\right\rangle_{I} & \mathbf{1}_{\mathcal{H}}
\end{array}\right)\left(\begin{array}{cc}
\mathbf{1}_{\mathcal{H}} & 0 \\
B^{*}(x) & \mathbf{1}_{\mathcal{H}}
\end{array}\right) \\
&=\left(\begin{array}{cc}
* & B(x)-\langle B\rangle_{I} \\
B^{*}(x)-\left\langle B^{*}\right\rangle_{I} & \mathbf{1}_{\mathcal{H}}
\end{array}\right) .
\end{aligned}
$$

Here, $\varrho$ denotes the spectral radius. In particular, $\left\|\left\langle W^{-1}\right\rangle_{I}^{1 / 2} W(x)\left\langle W^{-1}\right\rangle_{I}^{1 / 2}\right\|$ $\geq\left\|B(x)-\langle B\rangle_{I}\right\|$. 
This means that the matrix reverse Hölder condition 2.1, even with $r=2$, implies the norm BMO condition

$$
\sup _{I \subset \mathbb{R} \text { interval }} \frac{1}{|I|} \int_{I}\left\|B(x)-\langle B\rangle_{I}\right\| d x<\infty .
$$

However, it is known that boundedness of the commutator $[H, B]$ on $L^{2}(\mathbb{R}, \mathcal{H})$ does not imply that the norm BMO condition (21) for $B$ holds, since $\mathcal{L}(\mathcal{H})$ is not a UMD space (see [1]).

REMARK 5.2. We can give an easy concrete example for an operatorvalued $\mathcal{L}(\mathcal{H})$-valued function $B$ such that $[H, B]$ defines a bounded linear operator on $L^{2}(\mathbb{R}, \mathcal{H})$, but $B$ does not satisfy the norm BMO condition (21). This can be done by using the known fact that $l^{\infty}(\mathrm{BMO})$ (the space of uniformly bounded sequences of BMO functions) does not coincide with $\mathrm{BMO}_{\text {norm }}\left(l^{\infty}\right)$, the space of $l^{\infty}$-valued BMO-functions $\phi$ in the sense of uniform boundedness of the expressions

$$
\sup _{I \subset \mathbb{R} \text { interval }} \frac{1}{|I|} \int_{I}\left\|\phi(t)-m_{I} \phi\right\|_{\infty} d t
$$

(see also [2, Theorem 2.6] for the dyadic setting). Via (18), this yields a simple example of an operator weight as in 5.1.

Let $\left(e_{k}\right), k \in \mathbb{N}$, be an orthonormal basis of the infinite-dimensional separable Hilbert space $\mathcal{H}$ and let $e_{k} \otimes e_{k}$ be the corresponding rank 1 orthogonal projections.

Let $b: \mathbb{R} \rightarrow \mathbb{C}, b(t)=\chi_{[-1,1]}(t) \log |t|+1$. For $N \in \mathbb{N}, k \in\{0, \ldots, N\}$, let $b_{N, k}: \mathbb{R} \rightarrow \mathbb{C}, b_{N, k}(t)=b(t-k / N)$.

Let $B_{N}=\left(b_{N, 0}, \ldots, b_{N, N}\right)$. Then $\int_{-1}^{2} B_{N}(t) d t=0$ and $\left\|B_{N}(t)\right\|_{\infty} \geq$ $\log N$ for all $t \in(0,1)$, so

$$
\left\|B_{N}\right\|_{\mathrm{BMO}_{\mathrm{norm}}\left(l^{\infty}\right)} \geq \frac{1}{3} \log N, \quad \text { but } \quad\left\|B_{N}\right\|_{l^{\infty}(\mathrm{BMO})}=\|b\|_{\mathrm{BMO}}=C
$$

for all $N \in \mathbb{N}$ and some absolute constant $C$.

Via the isometric embedding $l^{\infty} \rightarrow \mathcal{L}(\mathcal{H}),\left(a_{k}\right) \mapsto \sum_{k=1}^{\infty} a_{k} e_{k} \otimes e_{k}$, we can think of the $B_{N}$ as operator-valued functions. In this case, $\left[H, B_{N}\right]$ is the block diagonal operator with the commutators $\left[H, b_{N, k}\right]$ on the diagonal, so its norm is equivalent to $\left\|B_{N}\right\|_{l^{\infty}(\mathrm{BMO})}$. On the other hand, the BMOnorm of $B_{N}$ in the sense of (21) is just $\left\|B_{N}\right\|_{\mathrm{BMO}_{\text {norm }}\left(l^{\infty}\right)}$. So (22) together with the formation of suitable infinite direct sums gives us the required counterexample.

Acknowledgements. The author thanks T. A. Gillespie for numerous fruitful discussions and Svetlana Roudenko for providing her with a preliminary unpublished version of the paper [8]. 


\section{References}

[1] O. Blasco, Hardy spaces of vector-valued functions: duality, Trans. Amer. Math. Soc. 308 (1988), 495-507.

[2] O. Blasco and S. Pott, Embeddings between operator-valued dyadic BMO spaces, Illinois J. Math., to appear.

[3] M. Christ and M. Goldberg, Vector $A_{2}$ weights and a Hardy-Littlewood maximal function, Trans. Amer. Math. Soc. 353 (2001), 1995-2002.

[4] T. A. Gillespie, S. Pott, S. Treil and A. Volberg, The transfer method in estimates for vector Hankel operators, Algebra i Analiz 12 (2000), no. 6, 178-193 (in Russian); English transl.: St. Petersburg Math. J. 12 (2001), 1013-1024.

[5] - , - - - - , Logarithmic growth for matrix martingale transforms, J. London Math. Soc. (2) 64 (2001), 624-636.

[6] - - - - - -, Logarithmic growth for weighted Hilbert transform and vector Hankel operators, J. Operator Theory 52 (2004), 103-112.

[7] T. A. Gillespie, S. Pott and J. Wilson, Matrix BMO and matrix $A_{2}$ weights: A substitute for the classical exp-relationship, Math. Proc. Cambridge Philos. Soc., to appear.

[8] M. Goldberg, Matrix $A_{p}$ weights via maximal functions, Pacific J. Math. 211 (2003), 201-220.

[9] R. Hunt, B. Muckenhoupt and R. Wheeden, Weighted norm inequalities for the conjugate function and Hilbert transform, Trans. Amer. Math. Soc. 176 (1973), $227-251$.

[10] F. Nazarov and S. Treil, The hunt for a Bellman function: applications to estimates for singular integral operators and to other classical problems of harmonic analysis, Algebra i Analiz 8 (1996), no. 5, 32-162 (in Russian); English transl.: St. Petersburg Math. J. 8 (1977), 721-824.

[11] M. C. Pereyra, Lecture notes on dyadic harmonic analysis, in: Contemp. Math. 289, Amer. Math. Soc., 2001, 1-60.

[12] S. Petermichl and S. Pott, A version of Burkholder's theorem for operator-weighted $L^{2}$-spaces, Proc. Amer. Math. Soc. 131 (2003), 3457-3461.

[13] S. Treil and A. Volberg, Wavelets and the angle between past and future, J. Funct. Anal. 143 (1997), 269-308.

[14] A. Volberg, Matrix $A_{p}$ weights via S-functions, J. Amer. Math. Soc. 10 (1997), $445-466$.

Department of Mathematics

University of Glasgow

Glasgow G12 8QW, UK

E-mail: sp@maths.gla.ac.uk 